Zugänge

Oper Orthop Traumatol 2020 · 32:200-208 https://doi.org/10.1007/s00064-020-00658-8 Eingegangen: 29. Juli 2019

Überarbeitet: 26. September 2019

Angenommen: 7. Oktober 2019

Online publiziert: 16. März 2020

(c) Der/die Autor(en) 2020

\section{Redaktion}

M. Mayer, München

Zeichnungen

R. Himmelhan, Mannheim

\section{Vorbemerkungen}

Das ventrale Release und die ventrale Fusion mit Verwendung von intervertebralen Cages hat sich als sehr potente und sichere Stabilisierungstechnik erwiesen $[1,2]$. Seit der Erstbeschreibung des ventralen Zugangs zur Lendenwirbelsäule durch Carpener 1932 [3] wurde dieser v. a. unter dem Aspekt der geringeren Invasivität bis hin zu einer echten minimalinvasiven Alternative weiterentwickelt. Mayer [4] beschrieb im Jahr 2000 den bis heute praktizierten und nachgewiesenermaßen komplikationsarmen anterolateralen retroperitonealen Zugang zur Lendenwirbelsäule (miniALIF [,anterior lumbar interbody fusion"]), der in neuerer Nomenklatur auch als OLIF („oblique lateral interbody fusion") bezeichnet wird. Im Unterschied zu dem lateralen transpsoatischen Zugang (XLIF [„extreme lateral lumbar interbody fusion"]) bedient sich der OLIF in einem anatomischen Korridor, der sich zwischen den großen abdominellen Gefäßen, die beim klassischen Mittellinienzugang (ALIF) präpariert werden müssen, und dem M. psoas, der den Plexus lumbalis enthält, befindet (• Abb. 1). Dieser Zugang hat auch bzw. v. a. seine Anwendung bei degenerativen Deformitäten gefunden und ist nicht nur bei monosegmentalen Pathologien, sondern auch multisegmental zur langstrecki-

Christoph Mehren $^{1,2} \cdot$ Lorenz Wanke-Jellinek $^{1,2} \cdot$ Alexander Krenauer $^{1,2}$. Andreas Korge ${ }^{1,2}$

'Wirbelsäulenzentrum, Schön Klinik, München Harlaching, München, Deutschland

${ }^{2}$ Akademisches Lehrkrankenhaus, Paracelsus Universität Salzburg, Salzburg, Österreich

\title{
Mono- und mehrsegmentaler ventraler minimalinvasiver Zugang zur Lendenwirbelsäule (OLIF) zur Korrektur von Deformitäten
}

gen Korrektur und Rekonstruktion der sagittalen Balance eine echte Alternative $\mathrm{zu}$ dorsalen Instrumentationen mit Korrekturosteotomien.

\section{Operationsprinzip und -ziel}

Darstellen des lateralen und ggf. auch ventralen Bandscheibenraumes L1-L5 (mit Erweiterung bis hin zu S1 möglich) über einen vorzugsweise linksseitigen, minimalinvasiven, muskelschonenden retroperitonealen Zugang. Durchführen einer ausgiebigen Diskektomie ggf. mit Resektion des vorderen Längsbandes, um ein maximales ventrales Release des Indexsegmentes zu erreichen. Anschließend interkorporelle Korrektur und Fusion mittels Cage und Knochenersatzmaterial bzw. autologem Knochenmaterial.

\section{Vorteile}

- Zugang zum Bandscheibenraum inklusive vorderem Längsband für ein anteriores Release

- Intervertebrale Relordosierungsund Korrekturmöglichkeit mittels entsprechender Implantate mit großer Auflagefläche

- Zugang über die konkave Seite der Krümmung über kleinen Zugang mehrsegmental möglich $(4-5 \mathrm{~cm})$
- Nutzung eines physiologischen Korridors zwischen abdominellen Gefäßen und M. psoas inklusive Plexus lumbalis

- Schonung des M. psoas mit geringem Risiko der Verletzung des Plexus lumbalis auch ohne den Einsatz von Neuromonitoring

- Erhaltung der Innervation der Bauchwandmuskulatur durch stumpfe Präparation jeweils in Muskelfaserrichtung

- Geringes iatrogenes Trauma mit geringem Blutverlust und niedriger Komplikationsrate

- Kurze Lernkurve

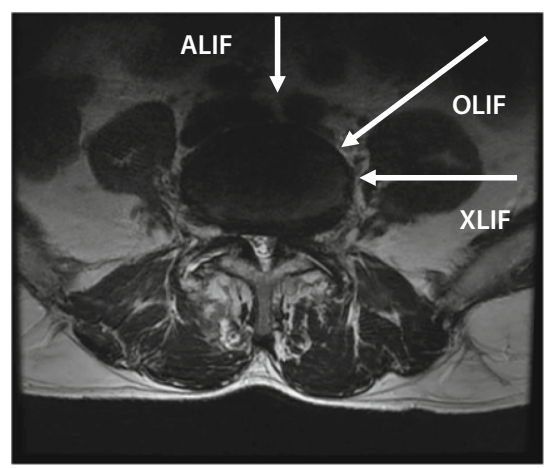

Abb. 1 A Schematische Darstellung der unterschiedlichen Zugangskorridore zur ventralen Lendenwirbelsäule. $A L I F$, anterior lumbar interbody fusion", OLIF "oblique lateral interbody fusion", XLIF "extreme lateral lumbar interbody fusion" 


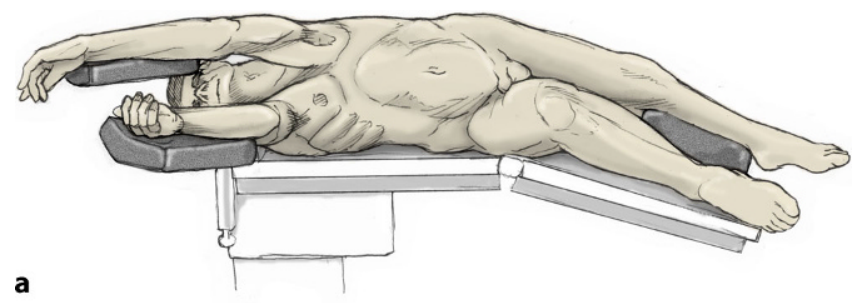

a

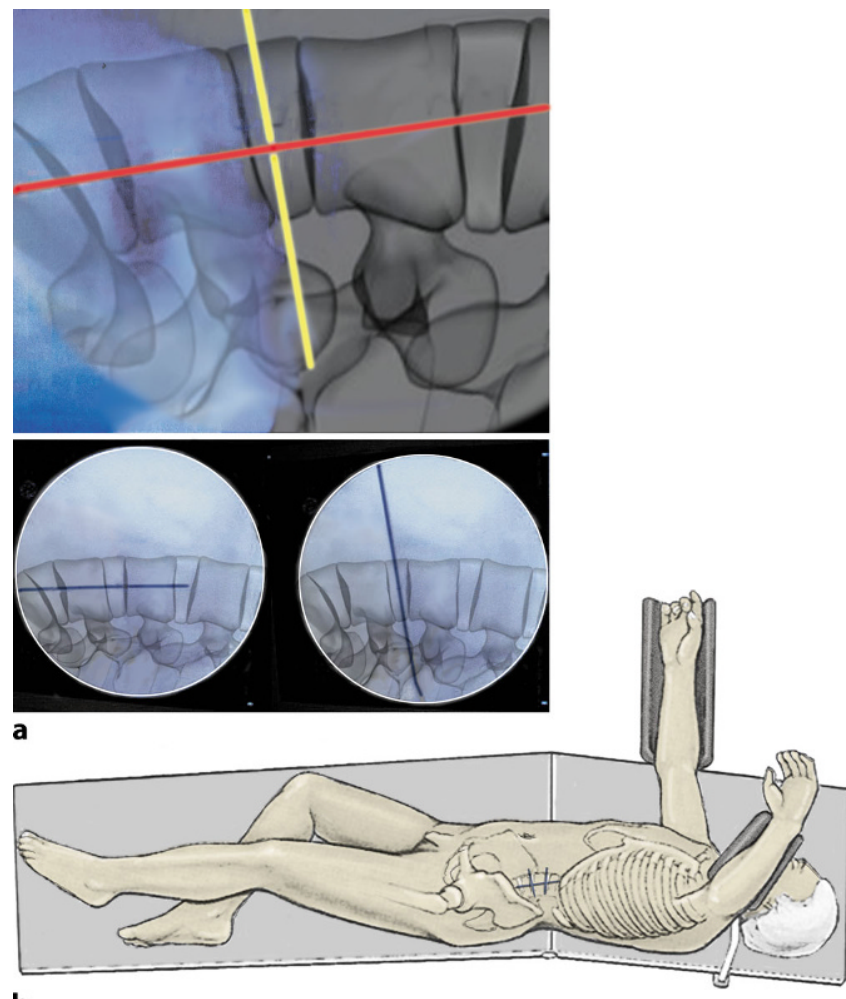

b

\section{Nachteile}

- Eingeschränkte Übersicht außerhalb des Zugangsgebietes

- Erschwerter Zugang bei hohem Beckenkamm v. a. zum Bandscheibenfach L4/5

- Erschwerter Zugang bei tiefem Rippenbogen v.a. zum Bandscheibenfach $\mathrm{L} 1 / 2$

- Kombinierter Zugang von L2 bis S1 über einen kleinen Zugang kaum möglich

- Erschwerte anatomische Verhältnisse v. a. bei ausgeprägter Rotation der Wirbelkörper

- Hochgradige Deformitäten erfordern ggf. eine ausgiebige Präparation der abdominellen Gefäße mit entsprechend erhöhtem Risiko einer Komplikation

- Zusätzlicher dorsaler Zugang zur additiven Instrumentation

- Lebensbedrohliche Komplikation bei Verletzung der abdominalen Gefäße (Aorta und V. cava) möglich

- Zugang über die konvexe Seite der Krümmung erfordert deutlich größeren Zugang bzw. getrennte Zugänge

- Rechtsseitiger Zugang erfordert ausgiebige Präparation der V. cava

\section{Indikationen}

- Mono- bzw. mehrsegmentales ventrales Release und interkorporelle Fusion zur Korrektur und Fusion bei degenerativen, posttraumatischen bzw. idiopathischen Deformitäten

- Für jede Altersgruppe möglich, insbesondere auch bei begleitender Osteoporose mit dem Vorteil einer großen Auflagefläche des Implantates

\section{Kontraindikationen}

Absolute Kontraindikationen bestehen nicht.

Relative Kontraindikationen sind gegeben bei:

- vorangegangenen Eingriffen im Retroperitoneum,

- retroperitonealer Fibrose (Morbus Ormond),
- Gefäßanomalien z. B. bei extrem lateralem Verlauf der V. iliaca communis bzw. Aortenaneurysmen,

- Fehlen von viszeral- bzw. gefäßchirurgischer Expertise zumindest als Back-up.

\section{Patientenaufklärung}

- Allgemeine Operationsrisiken (Infektion, Thrombose, Embolie, Nachblutung)

- Denervation des M. rectus abdominis bei Schädigung des N. iliocostalis

- Abdominelle Hernie aufgrund einer Fasziennahtinsuffizienz

- Druckläsionen des Plexus lumbalis durch zu kräftige Retraktion des M. psoas

- Leistenschmerz bzw. Gefühlsstörungen durch Verletzung des N. genitofemoralis

- Verletzung des sympathischen Grenzstranges mit Überwärmung des entsprechenden Beines aufgrund einer 
Oper Orthop Traumatol 2020 · 32:200-208 https://doi.org/10.1007/s00064-020-00658-8

(c) Der/die Autor(en) 2020

\section{Mehren · L. Wanke-Jellinek · A. Krenauer · A. Korge}

\section{Mono- und mehrsegmentaler ventraler minimalinvasiver Zugang zur Lendenwirbelsäule (OLIF) zur Korrektur von Deformitäten}

\section{Zusammenfassung}

Operationsziel. Minimalinvasiver anterolateraler Zugang zur Lendenwirbelsäule („oblique lateral interbody fusion" [OLIF]) zur Korrektur von lumbalen Deformitäten.

Indikationen. Ventrales Release bei degenerativer Lumbalskoliose oder segmentalen Kyphosen und interkorporelle Korrekturspondylodese.

Kontraindikationen. Absolute Kontraindikationen bestehen nicht. Relative Kontraindikationen sind vorangegangene (linksseitige) retroperitoneale Eingriffe bzw. Zustand nach Peritonitis mit ausgeprägten retroperitonealen Vernarbungen. Gefäßanomalien mit extrem lateral verlaufender V. iliaca communis (v. a. bei Segmentationsstörungen im vorletzten Bewegungssegment)

Operationstechnik. Über einen kleinen Hautschnitt im Bereich der linken Flanke und Wechselschnitttechnik durch die Bauchwandmuskulatur retroperitoneales Eingehen auf die laterale anteriore Lendenwirbelsäule monosegmental bzw. von L1-L5 Retraktion des M. psoas und Ausräumen des Bandscheibenfaches ggf. mit Durchtrennung des vorderen Längsbandes. Interkorporelles Release und Interposition eines Implantates zur ventralen Korrekturspondylodese. Weiterbehandlung. Frühmobilisation nach dorsaler Instrumentation nach Abklingen der Narkose unter Thromboseprophylaxe. Leichte Kost bis zum ersten Stuhlgang. Tragen einer rumpfstabilisierenden Orthese je nach Art und Ausdehnung des Eingriffes bis zu 12 Wochen. Keine Einschränkungen bezüglich Gehstrecke, Stehen und Sitzen unmittelbar postoperativ nach Schmerzvorgabe.

Ergebnisse. Es wurden 15 konsekutive Patienten mit degenerativer lumbaler Skoliose im Sinne einer ventrodorsalen Fusionsoperation versorgt. Die operative ventrale Versorgung beinhaltete 1 bis 4 Segmente. Erfasst wurden die zugangsspezifischen Komplikationen sowie die prä- und postoperativen radiologischen Parameter. Bei keinem der 15 linksseitig retroperitoneal durchgeführten ventralen Zugänge wurden intraoperative oder postoperative zugangsspezifische Komplikationen festgestellt. Im gesamten Patientenkollektiv konnte eine signifikante Reduktion des a.p.-Cobb-Winkels von präoperativ $16^{\circ} \pm 6^{\circ}$ auf $3^{\circ} \pm 2^{\circ}$ postoperativ $(p<0,001)$ erreicht werden.

\section{Schlüsselwörter}

Lumbalskoliose - Ventrale Fusion - Ventrales Release $\cdot$ Segmentale Kyphose · Interkorporelle Korrekturspondylodese

\section{The mono- and multisegmental ventral minimally invasive approach to the lumbar spine (OLIF) in lumbar deformities}

\section{Abstract}

Objective. Minimally invasive anterolateral approach to the lumbar spine (oblique lateral interbody fusion, OLIF) to correct lumbar deformities.

Indications. Ventral release in degenerative lumbar scoliosis or segmental kyphosis and intervertebral spondylodesis.

Contraindications. No absolute contraindications. Relative contraindications are previous (left-sided) retroperitoneal interventions or status after peritonitis with pronounced retroperitoneal scarring. Vascular anomalies with extremely lateral common iliac vein (especially with segmentation disorders in the penultimate mobile segment).

Surgical technique. Through a small skin incision in the left abdominal wall and alternating incision technique through the abdominal wall muscles retroperitoneal approach to the lateral anterior lumbar spine monosegmental or from L1-L5 multisegmental if needed. Retraction of the psoas muscle and removal of the intervertebral disc space, if necessary with resection of the anterior longitudinal ligament. Intervertebral release and interposition of an implant for ventral spondylodesis.

Postoperative management. Early mobilisation after dorsal instrumentation under thrombosis prophylaxis. Light meals until the first bowel movement. Wearing a trunk stabilizing brace for up to 12 weeks, depending on the type and extent of the procedure. No limitations regarding walking distance, standing and sitting immediately postoperatively.
Results. In all, 15 patients with degenerative lumbar scoliosis were treated with ventrodorsal fusion surgery. The surgical anterior treatment included 1-4 segments. The access-specific complications and preand postoperative radiological parameters were recorded. None of the 15 left-sided retroperitoneal ventral accesses showed intraoperative or postoperative access-specific complications. A significant reduction of the anteroposterior Cobb angle from $16^{\circ} \pm 6^{\circ}$ preoperatively to $3^{\circ} \pm 2^{\circ}$ postoperatively $(p<0.001)$ was achieved in the entire patient population.

\section{Keywords}

Lumbar scoliosis · Anterior fusion · Anterior release · Segmental kyphosis · Spinal fusion
Gefäßdilatation und ggf. Störung der Schweißsekretion

- Paralytischer Ileus

- Verletzung der großen abdominellen Gefäße (Aorta, V. cava, V. iliaca communis, V. lumbalis ascendens)

- Verletzung von Bauchorganen (Niere, Ureter, Milz, Darm)
- Duraverletzung bzw. Liquorfistel bei intraspinaler Präparation

- Implantatdislokation

- Postoperativer Korrekturverlust

- Pseudarthrose

\section{Operationsvorbereitungen}

- Röntgen der LWS (Lendenwirbelsäule) (ggf. auch Wirbelsäulenganzaufnahmen) in 2 Ebenen zur Bestimmung der Deformität, Bestimmung der sagittalen und koronaren Parameter, Orientierung und Höhe 
der Bandscheibenfächer, der topografischen Korrelation der Segmente zum Beckenkamm und Identifikation von lateralen Spondylophyten

- Magnetresonanztomographie der LWS zur Beurteilung des anatomischen Verlaufes der abdominellen Gefäße (inklusive Abklärung einer kaliberstarken V. lumbalis ascendens) und Beurteilung des Ausmaßes sowie Lagebeziehung des M. psoas zum anterolateralen Aspekt der LWS

- Bei einer schwierigen bzw. unklaren Gefäßanatomie ist die Anfertigung einer CT(Computertomographie)Angiographie der Abdominal- und Beckengefäße empfehlenswert.

- Bei zu erwartenden Schwierigkeiten bzw. erhöhtem Risiko einer Gefäßverletzung aufgrund der anatomischen Situation sollte bereits im Vorfeld der Kontakt zu einem Gefäßchirurgen hergestellt werden, um entweder den Zugang von Beginn an gemeinsam durchzuführen bzw. im notwendig werdenden Fall zeitnah Unterstützung zu erhalten.

- Bei ausgeprägten osteophytären Anbauten Computertomographie zum Ausschluss von Spontanfusionen bzw. Identifikation und Verlauf der lateralen Spondylophyten

- Gegebenenfalls Haarentfernung des Operationsgebietes kurz vor dem operativen Eingriff

- Am Vortag des Eingriffes leichte Kost mit rektalen Abführmaßnahmen

\section{Instrumentarium}

- Entsprechend lange Instrumente inklusive Fasszangen, Küretten, Meißel, Langenbeck-Haken, Bipolarkoagulation

- Gegebenenfalls selbsthaltende Retraktorensysteme

- Stirnlampe bzw. Lichtquelle an Retraktoren zur optimalen Ausleuchtung des Operationssitus

- Entsprechendes Implantatsystem für die anterolaterale Cageinterposition. Vor allem ovale Cagegeometrien mit Abstützung auf den beiden lateralen Apophysen eignen sich für diesen Zugang besonders gut. Auch hyperlordotische Cages können das radiologische Outcome positiv

beeinflussen.

- C-Bogen zur intraoperativen Höhenkontrolle bzw. Kontrolle der Implantatlage

- Gefäßchirurgisches Sieb als Stand-by

\section{Anästhesie und Lagerung}

- Intubationsnarkose, zentraler Venenkatheter, arterielle Druckmessung, Blasenkatheter, ggf. Magensonde

- Zur objektiven Überwachung der arteriellen Versorgung der entsprechenden Extremität kann ein Sättigungssensor an der Großzehe angebracht werden.

- Empfehlenswert ist eine Rechtsseitenlagerung auf einem kippbaren, röntgendurchlässigen Operationstisch (• Abb. 2a, b).

- Fluoroskopische Höhenlokalisation und topografische Projektion der entsprechenden Bandscheibenzentren auf die anterolaterale Bauchwand (- Abb. 3)

- Der Operateur steht dorsalseitig des Patienten, der Assistent ventralseitig. Die instrumentierende Pflegekraft steht neben dem Operateur, wobei der Instrumentiertisch über die Beine des Patienten geschoben wird.

\section{Operationstechnik}

- Abb. 4, 5, 6, 7

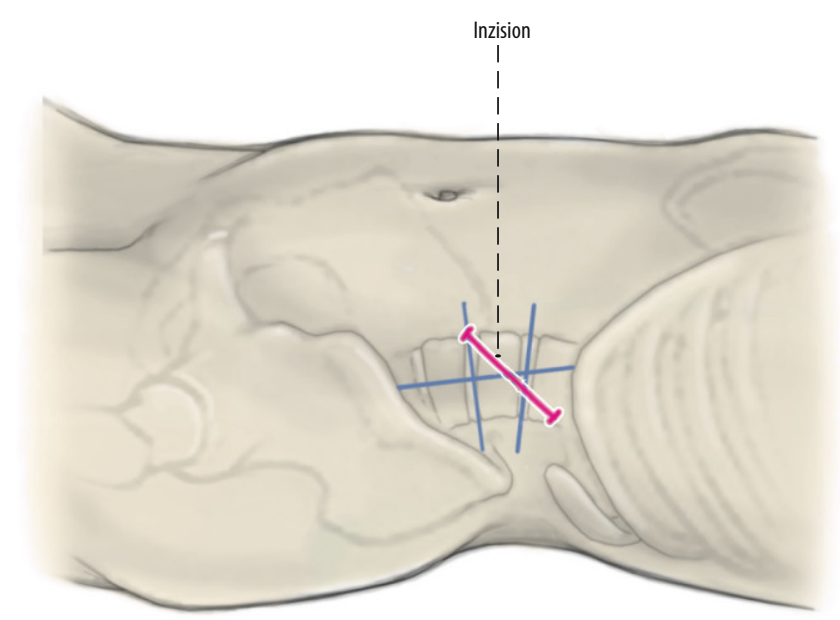

Abb. 4 ム Der Hautschnitt wird v. a. bei mehrsegmentalen Zugängen meist über dem zentralen Bandscheibenfach mit 5-8cm Länge (je nach Anzahl und Orientierung der Bandscheibenfächer) schräg im Faserverlauf des M. obliquus externus gesetzt. Scharfes Durchtrennen des subkutanen Fettgewebes bis auf die kräftige Faszie des M. obliquus externus unter Zuhilfenahme von Selbsthaltespreizern. (Mod. nach [7]) 


\section{Zugänge}
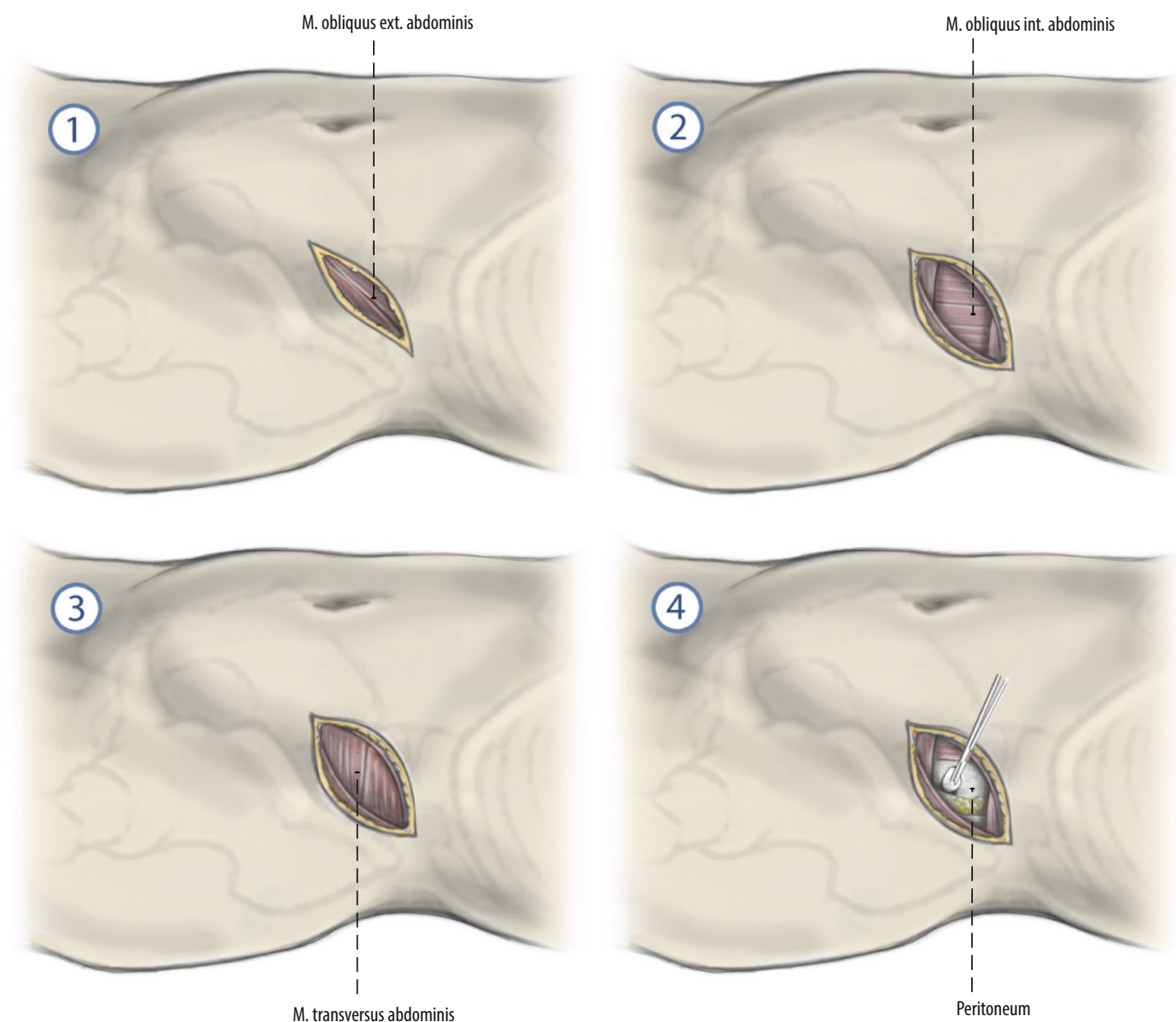

Abb. 5 Scharfes Eröffnen der ersten Muskelfaszie und stumpfe Präparation mit Präpariertupfer im Faserverlauf der jeweiligen Muskelschicht (1). Identisches Vorgehen durch den M. obliquus internus (2) und M. transversus abdominis (3) in Wechselschnitttechnik bis retroperitoneales Fettgewebe erscheint. Die Äste der Interkostalnerven sowie der N. iliohypogastricus/ ilioinguinalis können bei diesem Zugang v. a. zu den tieflumbalen Bandscheibenfächern gelegentlich das Operationsgebiet zwischen dem M. obliquus internus und M. transversus abdominis kreuzen. Diese sollten zur Sicherstellung der Innervation des M. rectus abdominis unbedingt durch stumpfes Abschieben erhalten werden. Zudem ist es empfehlenswert, den M. transversus abdominis möglichst von weit lateral nach medial zu dissezieren, um einer Verletzung des Peritoneums, das sich direkt an den M. transversus anlagern kann, vorzubeugen. Der Peritonealsack wird nun von dorsal her mobilisiert und nach ventral verlagert (4). (Mod. nach [7]) 


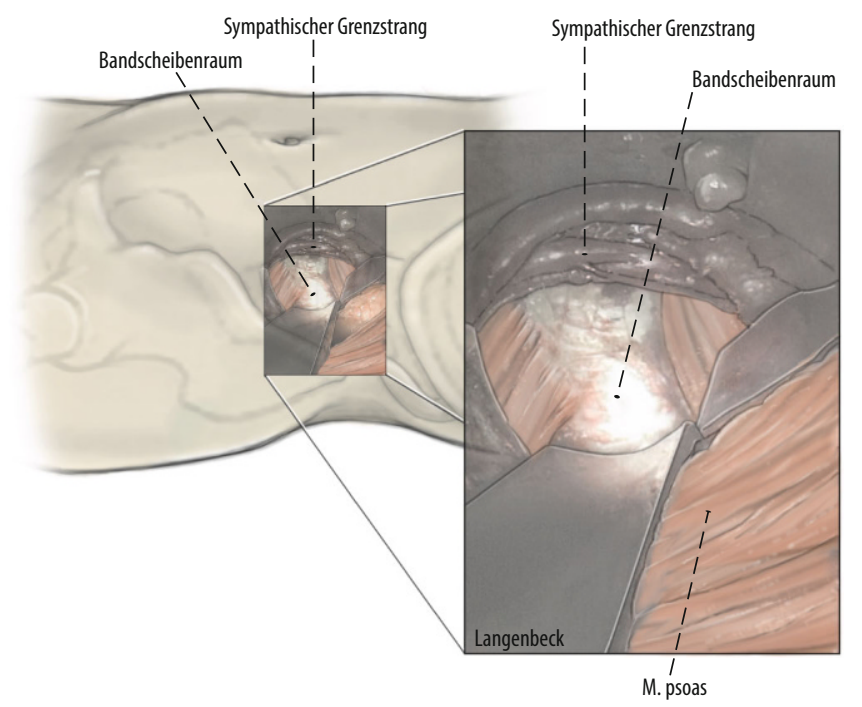

Abb. 6 \ Stumpfes Eingehen entlang des Retroperitonealraumes auf die ventrale Begrenzung des M. psoas. Identifikation der entsprechenden Bandscheibenräume durch Abschieben des M. psoas mithilfe von Langenbeck-Haken und Stielchen nach dorsal. Meist müssen kleinere Ansätze des Muskels an den angrenzenden Wirbelkörpern abgelöst werden, um eine ausreichende Retraktion zu erreichen. Direkt auf dem Bandscheibenraum finden sich kaum Insertionen des M. psoas, sodass hier eine orientierende Retraktion zu Beginn relativ einfach durchgeführt werden kann. Teilweise ist v. a. bei muskelkräftigen Patienten eine Koagulation von kleineren Blutungen aus dem M. psoas notwendig. Darstellen des vorderen Längsbandes als ventrale Landmarke. Die ventralen Strukturen wie V. cava, Aorta werden vorsichtig bei Bedarf von der ventralen Wirbelsäulenbegrenzung abgeschoben und ebenfalls mit einem Langenbeck-Haken aus dem Operationsfeld gehalten bzw. geschützt. Der Ureter wird ebenso mitsamt dem Peritonealsack nach ventral gehalten. Besondere Vorsicht ist v. a. im Umgang mit den abdominellen Gefäßen bei der Präparation des Segmentes L4/5 geboten, da es bei einer hohen Bifurkation der V. cava zu einem sehr lateralen Verlauf und einer Überdeckung des Bandscheibenraumes mit der V. iliaca communis kommen kann. Selten existiert auch eine kaliberstarke V. lumbalis ascendens, die ebenfalls eine Medialisierung des Gefäßbündels einschränkt. Bei Bedarf kann diese folgenlos unterbunden und durchtrennt werden. Nach Darstellen der lateralen Bandscheibenzirkumferenz sollte der Versuch unternommen werden, den sympathischen Grenzstrang nach ventral zu mobilisieren. Bei eher kranialen Segmenten kann eine Mobilisation nach dorsal ebenso erfolgen. Sollte dies nicht möglich sein, können eine Kauterisation und Durchtrennung über dem entsprechenden Bandscheibenraum notwendig sein. Im überwiegenden Anteil der Fälle hat dies keine wesentlichen Auswirkungen auf den Patienten. Der N. genitofemoralis verläuft am ventralen Rand des M. psoas. Dieser sollte zur Vermeidung von Parästhesien sowie Schmerzen im Bereich der Leiste geschont werden. Vor allem bei lumbalen Skoliosen mit entsprechender Rotation der Wirbelkörper können die anatomischen Strukturen deutlich verlagert sein. Hier sollte besonderes Augenmerk auf den individuellen anatomischen Verlauf v. a. der Gefäße gerichtet werden. Bei mehrsegmentalem Vorgehen ist eine Durchtrennung der Segmentgefäße nicht notwendig, da diese Prozedur schrittweise Bandscheibenraum für Bandscheibenraum wiederholt wird, ohne eine durchgehende Retraktion des M. psoas durchzuführen

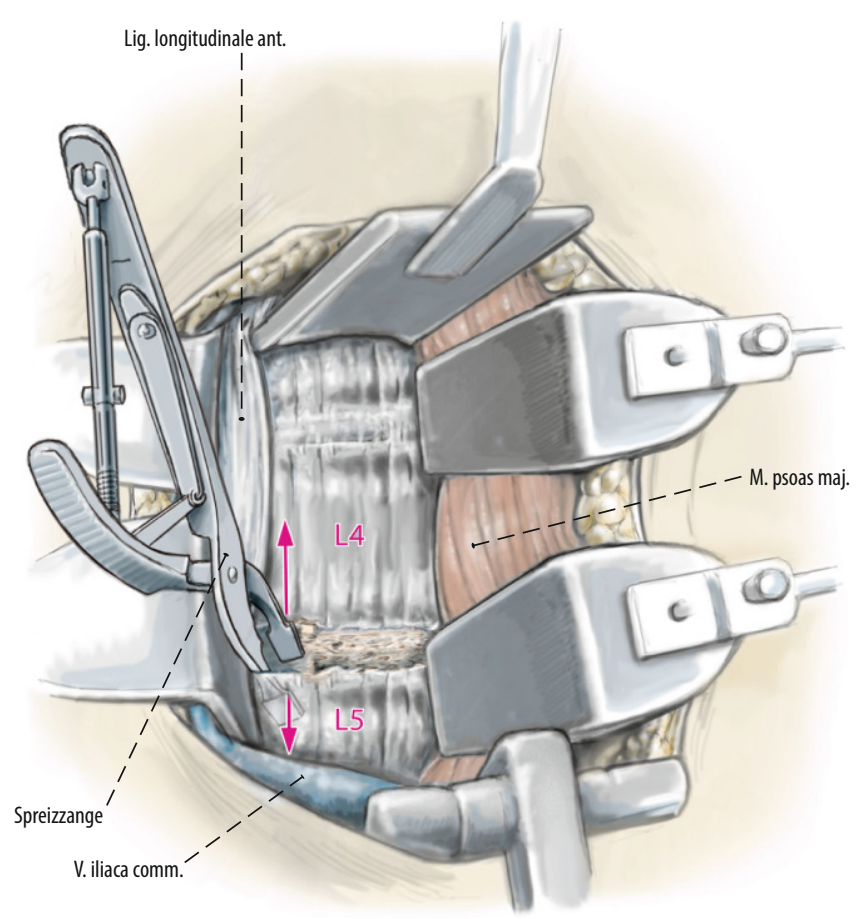

Abb. 7 \ Darstellung des anterolateralen Aspektes des entsprechenden Bandscheibenraumes und Durchführen einer subtotalen Diskektomie. Gegebenenfalls müssen laterale Osteophyten mit Meißeln entfernt werden. Sollte die räumliche Orientierung des Bandscheibenraumes Schwierigkeiten bereiten, ist es ratsam, diese mit einem erneuten fluoroskopischen Bild zu verifizieren, um eine Zerstörung der Endplatten zu vermeiden. Mithilfe von intervertebralen Distraktoren kann ein ausgedehntes ventrales Release bis hin zur kontralateralen Bandscheibenbegrenzung mit Resektion des kontralateralen Anulus v. a. bei hochgradigen Deformitäten komplettiert werden. Kürettage der Grund- und Deckplatte zur vollständigen Entknorpelung derselben. Interposition eines entsprechenden Implantates, das mit Fusionsmaterial gefüllt wurde. Abdecken des Bandscheibenraumes mit einem Hämostyptikum. Die Einlage einer Drainage ist in der Regel nicht notwendig. Abschließende Reposition des Peritonealsackes und fortlaufende Naht der einzelnen Muskelfaszien. Subkutannaht, Hautdesinfektion und resorbierbare Intrakutannaht. Steriler Wundverband. (Mod. nach [7]) 


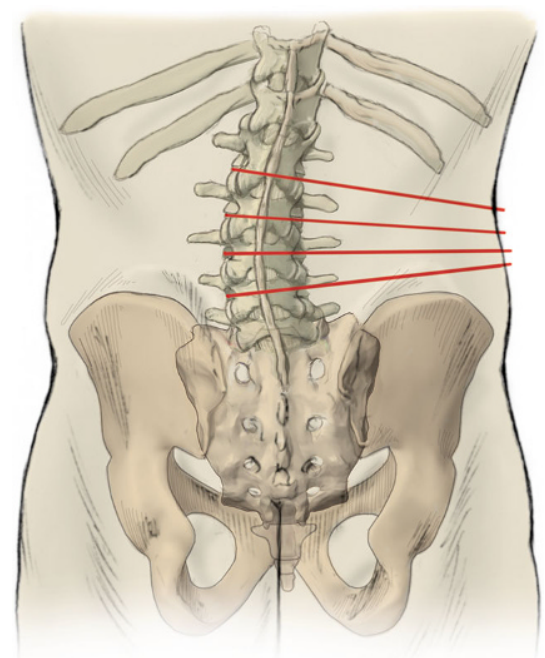

Abb. 8 A Schematische Darstellung der Planung der Schnittführung mit dem Vorteil des deutlich kleineren Zugangs über die konkave Seite

\section{Besonderheiten}

Bei der operativen Behandlung von lumbalen Skoliosen ist prinzipiell ein Zugang über die konvexe bzw. konkave Seite der Krümmung möglich. Prinzipiell ist eine linksseitige retroperitoneale Präparation auf die Wirbelsäule technisch deutlich einfacher bzw. komplikationsärmer, da die V. cava bis zur Bifurkation rechts mediolateral an der Wirbelsäule entlangläuft und der Korridor zwischen der Vene und dem M. psoas meist deutlich kleiner ist. Dies bedeutet, dass in der Mehrzahl der Fälle eine Mobilisation der V. cava notwendig wäre. Prinzipiell hat das Eingehen über die konkave Seite der Krümmung im Gegensatz zu der konvexen Seite den Vorteil, dass aufgrund der „fächerförmigen“ Anordnung der Bandscheibenräume ein zentraler kleiner Zugang gewählt werden kann (• Abb. 8). Zudem hat man einen direkten Zugang zu evtl. vorhandenen osteophytären und teilweise überbrückenden Anbauten, die sich bei degenerativen Skoliosen häufig an der konkaven Seite ausbilden. Bei ausgeprägten Krümmungen kann ein Eingehen über die konvexe Seite sogar getrennte $\mathrm{Zu}$ gänge zu den entsprechenden Segmenten notwendig machen. Nichtsdestotrotz ist die persönliche Empfehlung der Au-
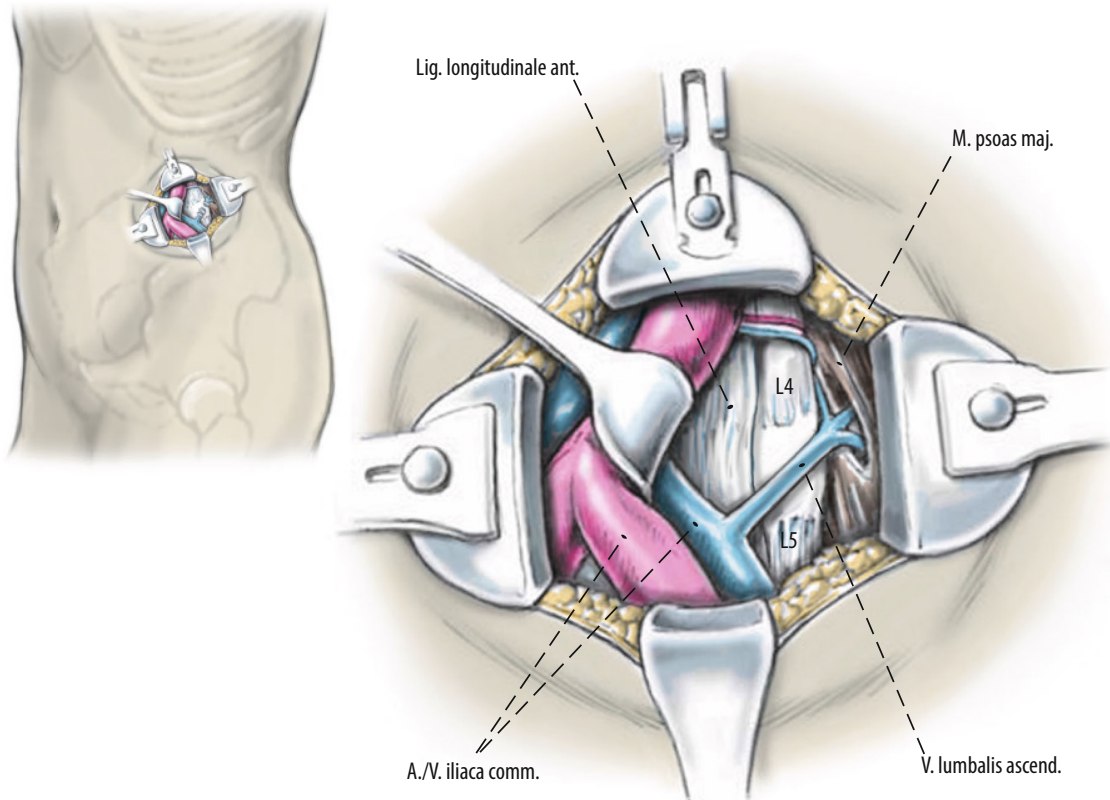

Abb. 9 A Mobilisation und Protektion des Gefäßbündels sowie Darstellung des Lig. longitudinale anterius, das v. a. bei Kyphosen oder hochgradigen Lordosekorrekturen durchtrennt werden sollte. (Aus [7])

toren, standardmäßig einen linksseitigen retroperitonealen Zugang aufgrund des geringeren Komplikationspotenzials $\mathrm{zu}$ favorisieren.

Sollte eine Kyphose oder sehr rigide Abflachung der Lendenwirbelsäulenlordose die Resektion des vorderen Längsbandes notwendig machen, ist es ratsam, nach vorsichtigem Lösen des Gefäßbündels einen Langenbeck-Haken bzw. einen Spatel zwischen Gefäßen und Längsband zu platzieren, um einer akzidentellen Verletzung der dorsalen Gefäßwand vorzubeugen (• Abb. 9).

\section{Postoperative Behandlung}

- Thromboseprophylaxe mit fraktioniertem Heparin bis zur vollständigen Mobilisation

- Frühmobilisation am 1. postoperativen Tag ohne Einschränkungen von Sitzen, Gehen und Stehen

- Gegebenenfalls stabilisierende Rumpforthese je nach Art des Eingriffs und additiver dorsaler Versorgung bis zu 12 Wochen nach individuellem Versorgungsstandard für langstreckige lumbale Fusionen

- Antibiotische Abdeckung (z. B. Cefuroxim $1,5 \mathrm{~g}$ i.v.) nach Hausstandard perioperativ
- Kostaufbau bzw. leichte Kost bis zum ersten Stuhlgang

- Primärer Pflasterverband für $48 \mathrm{~h}$ postoperativ, dann je nach Befund

- Röntgenkontrolle in 2 Ebenen bei entsprechender Mobilisation im Stehen postoperativ sowie nach 3 und 12 Monaten

- Die Arbeitsfähigkeit ist je nach beruflicher Belastung und individuellem Heilverlauf nach 6 bis 12 Wochen in der Regel wieder gegeben.

\section{Fehler, Gefahren, Komplikationen}

- Falsche Lagerung des Patienten: Durch ungenügendes „Aufklappen“ des Patienten kann es zu einer Überlagerung des Bandscheibenfaches L4/5 durch den Beckenkamm sowie L2/3 durch den Rippenbogen kommen. Dies erhöht die Gefahr, das Bandscheibenfach nicht in der notwendigen Orientierung ausräumen und mit einem Implantat bestücken zu können, was zu einer Verletzung der Grund- bzw. Deckplatte führt und ein Sintern des Implantates postoperativ begünstigt. Durch entsprechende Positionierung des Patienten unter seitlicher BV(Bildverstärker)-Kon- 
trolle sollte der nahezu senkrechte Zugang zum Bandscheibenraum ggf. auch intraoperativ identifiziert und sichergestellt werden. Bei Verletzung der Wirbelkörperendplatten sollte der Defekt durch ein entsprechend großes Implantat überbrückt werden.

- Ungenügendes Release/Implantatsinterung: Die Präparation und Durchtrennung des vorderen Längsbandes ist v. a. bei der Rekonstruktion der LWS-Lordose ein entscheidender und präparatorisch kritischer Punkt. Durch eine unzureichende Mobilisation der ventralen Bandscheibenanteile steigt das Risiko einer postoperativen Implantatsinterung mit postoperativem Korrekturverlust. Vor allem bei herabgesetzter Knochenqualität sollte ein ausreichendes Release mit Durchtrennung des vorderen Längsbandes sichergestellt werden. Zudem sollten die Implantate eine größtmögliche Auflagefläche bieten und idealerweise von der ipsilateralen bis zur kontralateralen Apophyse reichen, um postoperative Sinterungen zu vermeiden. Dies ist bei der Verwendung von hyperlordotischen Implantaten von noch größerer Bedeutung.

- Vor allem durch eine Deformität mit entsprechender Rotation der Wirbelkörper kann es zu einer deutlichen Verlagerung der Gefäße kommen und eine Gefäßpräparation notwendig werden. Sollte es zu einer Verletzung der großen abdominellen Gefäße kommen, ist unverzüglich auf eine gefäßchirurgische Expertise zurückzugreifen, ohne einen höheren Blutverlust durch ein eigenständiges Manöver zu riskieren. Bis zum Eintreffen eines Gefäßchirurgen können venöse Blutungen gut über eine entsprechende Tamponade kontrolliert werden. Sollte es zu einer arteriellen Verletzung kommen, ist eine kontrollierte Kompression mittels Stiels Erfolg versprechend. Parallel sollten entsprechende Vorbereitung getroffen werden (Cell-Saver, Kreuzblut, Konserven, Zugänge etc.).

\section{Ergebnisse}

In einer von uns durchgeführten, retrospektiven Studie inkludierten wir $15 \mathrm{~Pa}$ tienten (12 Frauen, 3 Männer) mit degenerativer lumbaler Skoliose, die im Kalenderjahr 2018 mit einem operativen Eingriff im Sinne einer ventrodorsalen Fusionsoperation versorgt wurden.

Die inkludierten Patienten waren zwischen 34 und 84 Jahre alt mit einem medianen Alter von 71 Jahren. Die operative ventrale Versorgung beinhaltete 1 bis 4 Segmente. Erfasst wurden die zugangsspezifischen Komplikationen sowie die prä- und postoperativen radiologischen Parameter. Um die Rekonstruktion der sagittalen Balance und Besserung der degenerativen Deformität beurteilen zu können, erfolgte die Ausmessung von Parametern der sagittalen Balance (lumbale Lordose [L1-S1], Pelvic-Inzidenz [PI], Sacral-Slope [SS]) sowie des a.p.-Cobb Winkels des operativ versorgten Bereichs anhand von radiologischen Aufnahmen.

Insgesamt wurden im Sinne einer ventrodorsalen Prozedur 35 Segmente versorgt. Bei keinem der 15 linksseitig retroperitoneal durchgeführten ventralen Zugänge wurden intraoperative wie auch postoperative zugangsspezifische Komplikationen festgestellt.

Die Auswertung der Messungen ergab eine präoperative durchschnittliche Pelvic-Inzidenz von $57^{\circ}$. Postoperativ kam es zu einer leichten Zunahme der lumbalen Lordose (L1-S1) des gesamten Patientenkollektiv von $41,5^{\circ} \pm 11,3^{\circ}$ präoperativ auf $43,5^{\circ} \pm 10,5^{\circ}$. Betrachtete man die Gruppe von Patienten $(n=10)$, die aufgrund präoperativer fehlender Lordose mit hyperlordotischen Cages $\left(>10^{\circ}\right)$ versorgt wurden, so fand sich eine statistisch signifikante Zunahme der lumbalen Lordose von $38,6^{\circ} \pm 11,7^{\circ}$ präoperativ auf $45,2^{\circ} \pm 10,9^{\circ}$ postoperativ $(p<0,05)$. Zudem fand sich im gesamten Patientenkollektiv eine signifikante Reduktion des a.p.-Cobb-Winkels von präoperativ $16^{\circ} \pm 6^{\circ}$ auf $3^{\circ} \pm 2^{\circ}$ postoperativ $(p<0,001)$. Der gemessene SacralSlope zeigte einen nichtsignifikanten Anstieg von präoperativ $32,9^{\circ} \pm 7^{\circ}$ auf $34^{\circ} \pm 10^{\circ}$ postoperativ. Vergleichbare Ergebnisse konnten in klinischen und biomechanischen Studien nachgewiesen werden [5]. Zudem konnte gezeigt werden, dass durch die OLIF-Technik sich auch klinische Parameter wie VAS (visuelle Analogskala) und ODI (Oswestry Disability Index) signifikant postoperativ verbessern ließen [6].

\section{Korrespondenzadresse}

\section{Dr. Christoph Mehren}

Wirbelsäulenzentrum, Schön Klinik, München Harlaching

Harlachinger Str. 51, 81547 München,

Deutschland

CMehren@schoen-klinik.de

Funding. Open access funding provided by Paracelsus Medical University.

\section{Einhaltung ethischer Richtlinien}

Interessenkonflikt. C. Mehren, L. Wanke-Jellinek, A. Krenauer und A. Korge geben an, dass kein Interessenkonflikt besteht.

Für diesen Beitrag wurden von den Autoren keine Studien an Menschen oder Tieren durchgeführt. Für die aufgeführten Studien gelten die jeweils dort angegebenen ethischen Richtlinien.

Open Access. Dieser Artikel wird unter der Creative Commons Namensnennung 4.0 International Lizenz veröffentlicht, welche die Nutzung, Vervielfältigung, Bearbeitung, Verbreitung und Wiedergabe in jeglichem Medium und Format erlaubt, sofern Sie den/die ursprünglichen Autor(en) und die Quelle ordnungsgemäß nennen, einen Link zur Creative Commons Lizenz beifügen und angeben, ob Änderungen vorgenommen wurden.

Die in diesem Artikel enthaltenen Bilder und sonstiges Drittmaterial unterliegen ebenfalls der genannten Creative Commons Lizenz, sofern sich aus der Abbildungslegende nichts anderes ergibt. Sofern das betreffende Material nicht unter der genannten Creative Commons Lizenz steht und die betreffende Handlung nicht nach gesetzlichen Vorschriften erlaubt ist, ist für die oben aufgeführten Weiterverwendungen des Materials die Einwilligung des jeweiligen Rechteinhabers einzuholen.

Weitere Details zur Lizenz entnehmen Sie bitte der Lizenzinformation auf http://creativecommons.org/ licenses/by/4.0/deed.de.

\section{Literatur}

1. Brau S (2002) Mini-open approach to the spine for anterior lumbar interbody fusion: description of the procedure, results and complications. Spine J 2:216-223. https://doi.org/10.1016/ S1529-9430(02)00184-5

2. Mehren C, Mayer HM, Zandanell C et al (2016) The oblique anterolateral approach to the lumbar spine provides access to the lumbar spine with few early complications. Clin Orthop Relat Res 
Buchbesprechung

474:2020-2027. https://doi.org/10.1007/s11999016-4883-3

3. Carpener N (1932) Spondylolisthesis. Br J Surg 19:374-386

4. Mayer HM (2000) The ALIF concept. Eur Spine J 9(Suppl 1):S35-S43

5. Ohtori S, Mannoji C, Orita S et al (2015) Mini-open anterior retroperitoneal lumbar interbody fusion: oblique lateral interbody fusion for degenerated lumbar spinal kyphoscoliosis. Asian Spine J 9:565-572. https://doi.org/10.4184/asj.2015.9.4. 565

6. Ohtori S, Orita S, Yamauchi Ket al (2015) Mini-open anterior retroperitoneal lumbar Interbody fusion: oblique lateral Interbody fusion for lumbar spinal degeneration disease. Yonsei Med J56:1051-1059. https://doi.org/10.3349/ymj.2015.56.4.1051

7. Mehren CH, Mayer M, Siepe C, Grochulla F, Korge A (2010) Der minimalinvasive anterolaterale Zugang zu L2-L5. Oper Orthop Traumatol 22:221-228

H. Dittrich, M. Schimmack, C.-H. Siemsen

\section{Orthopädische Biomechanik}

Einführung in die Endoprothetik der Gelenke der unteren Extremitäten

Springer Vieweg 2019, 1., 186 S., 326 Abb., (ISBN: 978-3-662-55332-9), Softcover 37,99 EUR

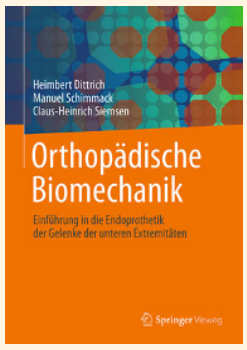

Die Biomechanik beschäftigt sich mit der Betrachtung biologischer Systeme unter Verwendung von Methoden und Gesetzmäßigkeiten der technischen Mechanik.

Dies verdeutlicht bereits die Bedeutung des Zusammenführens verschiedener wissenschaftlicher Disziplinen unter Berücksichtigung technisch-physikalischer, anatomischbiologischer und medizinischer Aspekte.

Dieser so wichtige und gleichzeitig so faszinierende Grundsatz der interdisziplinären Betrachtung biomechanischer Problemstellungen wurde von den Autoren dieses Buches in hervorragender und konsequenter Weise umgesetzt. Daher eignet es sich als grundlegende Lektüre für eine große Gruppe biomechanisch Interessierter wie zum Beispiel Studierende der Medizin oder Medizintechnik aber auch für Angehörige verwandter Berufsgruppen der Orthopädie, Unfallchirurgie, Endooprothetik, Rehabilitation, Physiotherapie oder Orthopädietechnik. Diesem Anspruch wird das Buch gerecht, weil für den Leser medizinisch-anatomische Grundlagen des Haltungs- und Bewegungsapparates, auf welche dieses Buch fokussiert, in ansprechender und anschaulicher Weise mit der technischen Mechanik und dabei strukturiert und logisch im Aufbau sowie unterstützt durch zahlreiche eindrückliche Bilder, Tabellen und Graphiken vermittelt.
Insgesamt eignet sich die "Orthopädische Biomechanik" der Autoren Dittrich, Schimmack und Siemsen in besonderer Weise nicht nur für den Einsatz in der Lehre, sondern auch als Nachschlagewerk beispielsweise in der biomechanischen oder orthopädischtraumatologischen täglichen Praxis.

F. Lampe 